\title{
Optical properties and refractive index sensitivity of reactive sputtered oxide coatings with embedded Au clusters
}

\author{
N. M. Figueiredo, ${ }^{1,2, a)}$ T. Kubart, ${ }^{2}$ J. A. Sanchez-García, ${ }^{3}$ R. Escobar Galindo, ${ }^{4}$ \\ A. Climent-Font, ${ }^{5}$ and A. Cavaleiro ${ }^{1}$ \\ ${ }^{1}$ SEG-CEMUC - Department of Mechanical Engineering, University of Coimbra, Rua Luís Reis Santos, \\ 3030-788 Coimbra, Portugal \\ ${ }^{2}$ The Angström Laboratory, Uppsala University, P.O. Box 534, SE-751 21 Uppsala, Sweden \\ ${ }^{3}$ Energy and Environment Division, TECNALIA, San Sebastian 20009, Spain \\ ${ }^{4}$ Instituto de Ciencia de Materiales de Madrid, ICMM-CSIC, Campus Cantoblanco, 28049 Madrid, Spain \\ ${ }^{5}$ Centro de Micro-Análisis de Materiales, Universidad Autónoma de Madrid, 28049 Madrid, Spain
}

(Received 31 August 2013; accepted 16 December 2013; published online 14 February 2014)

\begin{abstract}
In the present study, nanocomposite coatings of Au clusters embedded in two different oxides, $\mathrm{TiO}_{2}$ and $\mathrm{Al}_{2} \mathrm{O}_{3}$, were synthesized using pulsed $\mathrm{DC}$ magnetron sputtering. The depositions were carried out in three steps, by depositing the oxide, the Au clusters, and again the oxide. The deposition time of the Au clusters was varied in order to achieve different cluster sizes, morphologies, and nanocomposite topographies. The structure, microstructure, morphology, and the optical properties of the coatings were studied. With the increase in Au content, red-shifted surface plasmon resonance (SPR) peaks with higher intensity and increased widths were observed due to changes in the metal clusters sizes and morphology and due to interparticle effects. In order to relate the peculiar SPR extinction bands with the different clusters shapes and distributions, a simulation of the optical properties of the nanocomposites was performed making use of the Renormalized Maxwell-Garnett approach. A theoretical study concerning the refractive index sensitivity was made in order to predict the optimal coatings parameters for sensing experiments. The increased surface area and the strong SPR extinction bands make these coatings suitable for gas sensing and also catalysis, albeit many other application fields can be envisaged. ( 2014 AIP Publishing LLC.

[http://dx.doi.org/10.1063/1.4861136]
\end{abstract}

\section{INTRODUCTION}

Thin composite films consisting of metal nanoparticles in an oxide matrix are of interest for applications in electronics, sensors, catalysis, and biology. ${ }^{1}$ This increasingly interest is mainly due to the exhibition of Localized Surface Plasmon Resonances (LSPRs) by the nanoparticles, optically induced oscillations of free electrons at the surface of the noble metal nanoparticles. The excitation of LSPRs results in strong light extinction effects, which are heavily dependent on the nanoparticle's dielectric constant, size, and shape but also on the dielectric constant of the surrounding medium. ${ }^{2,3}$ Materials exhibiting LSPR are thus attractive for a number of applications, including gas sensors.

When metallic clusters exhibiting LSPR at a suitable wavelength are embedded in chemically reactive dielectric matrixes, significant change in the optical properties of the nanocomposites (e.g., reflectivity) can occur as a result of the interaction between the metal oxide and the gas molecules (either by chemical reaction or by physical adsorption). Since the LSPR is highly dependent on the dielectric properties of both the nanoparticles and the surrounding medium, molecule-induced changes in the vicinity of the nanoparticles can be detected by simply monitoring the position of the LSPR extinction peak. The detection of small

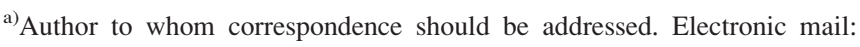
nuno.figueiredo@dem.uc.pt.
}

concentrations of gas molecules can thus be carried out in a novel way. ${ }^{4,5}$

Optical sensors have several inherent advantages as compared to other sensor technologies including the ability to monitor different optical properties of the sensing material (transmission, reflection, luminescence, etc.), compatibility with optical fibre based remote sensing approaches and the multi-gas detection capability (by using differences in the intensity, wavelength, phase, and polarization of the output light signals). They also offer more robust operation thanks to higher resistance to electromagnetic noise than conventional resistive sensors. The LSPR gas sensors further allows simultaneous temperature and gas sensing by monitoring of the SPR peak width and position. ${ }^{6-8}$

The short (and tunable) characteristic electromagnetic field decay length is responsible for the enhanced sensitivity of the LSPR nanosensor. ${ }^{9}$ Moreover, the LSPR nanosensors possess smaller footprint and cost at least 30-60 times less than commercialized thin film SPR sensors. ${ }^{3}$ The addition of noble metals in the form of nanoparticles can enhance the response of many metal oxides to different gases by increasing the active surface area, improving the gas diffusion inside the film, and allowing, in certain cases, the selective adsorption of the target gas. LSPR sensors can thus be a suitable option for sensing in control and measuring systems with excellent performance, reliability, and low price. ${ }^{10}$

Nanostructured materials exhibiting LSPR have been prepared mostly by colloid chemical methods, vacuum 
evaporation, lithographic techniques, laser ablation, and electrodeposition. ${ }^{11}$ Among all, the technique of magnetron sputtering has been widely used due to its low cost, simplicity, and versatility. ${ }^{12}$

In this study, nanostructured coatings suitable for refractive index LSPR gas sensors were deposited by alternating sputtering. The coatings consisted of nanoclusters of $\mathrm{Au}$ embedded in a $\mathrm{TiO}_{2}$ or $\mathrm{Al}_{2} \mathrm{O}_{3}$ matrix and followed a simple but effective design, carefully chosen to allow optimization of the light extinction and the overall gas adsorption of the system.

$\mathrm{Au}$ is one of the most inert materials known today, and it exhibits LSPR in the visible light spectrum. $\mathrm{TiO}_{2}$ has many possible applications such as in photo-catalysis, heterogeneous catalysis, as gas sensors, solar cells, and bio-compatible materials; ${ }^{13}$ as a gas sensor, $\mathrm{TiO}_{2}$ is sensitive to $\mathrm{O}_{2}, \mathrm{H}_{2}, \mathrm{CO}, \mathrm{CH}_{4}$, and to several alcohols. ${ }^{14} \mathrm{Al}_{2} \mathrm{O}_{3}$ is widely used in mechanical, optical, and micro-electronic applications because of its excellent chemical resistance, good mechanical strength, high hardness, transparency, high abrasive, and corrosion resistance as well as high insulating strength. ${ }^{15} \mathrm{Al}_{2} \mathrm{O}_{3}$ is an excellent sensing material for humidity, and sensors based on $\mathrm{Al}_{2} \mathrm{O}_{3}$ operate mainly at near room temperature making use of porous microstructures. At higher temperatures, they can also sense $\mathrm{H}_{2}, \mathrm{CO}_{2}$, and $\mathrm{O}_{2} .{ }^{14}$

The deposited nanocomposite thin films of $\mathrm{Au} @ \mathrm{TiO}_{2}$ and $\mathrm{Au} @ \mathrm{Al}_{2} \mathrm{O}_{3}$ were characterized with respect to the structure, microstructure, surface morphology, and optical properties. Results of this characterization are summarized in Sec. IV.

Then, a theoretical analysis of the nanocomposite's optical response was performed in order to identify coating parameters that allow maximizing their sensing performance. These results are presented in Sec. V.

\section{EXPERIMENTAL DETAILS}

Experiments were performed in a Lesker CMS-18 deposition system equipped with four magnetron sputtering sources.

$\mathrm{Ti}$ and $\mathrm{Al}$ targets $(99.995 \%$ purity) with a thickness and diameter of 6 and $100 \mathrm{~mm}$, respectively, were used for depositing $\mathrm{TiO}_{2}$ and $\mathrm{Al}_{2} \mathrm{O}_{3}$ under reactive atmosphere. $\mathrm{Au}$ target (99.995\% purity) with a thickness and diameter of 3 and $50 \mathrm{~mm}$, respectively, was utilized for depositing the nanoparticles. Au was deposited using a magnetron Torus $2 \mathrm{HV}$ by Kurt J. Lesker equipped with high strength magnetic assembly.

Base pressure of the system was below $3 \times 10^{-5} \mathrm{~Pa}$. The depositions were carried out at constant pressure by using a pressure-controlled gate valve in the pumping system. Au layers were deposited at a constant pressure of $1 \mathrm{~Pa}$ using 40 sccm of Ar. For the oxide layers, $15 \mathrm{sccm}$ of $\mathrm{O}_{2}$ was introduced in the chamber along with the $40 \mathrm{sccm}$ of Ar, and the deposition pressure was set to $0.5 \mathrm{~Pa}$. All depositions were carried out in pulsed DC mode with a Pinnacle Plus Advanced Energy generator. The Au was deposited at a constant power of $65 \mathrm{~W}$, frequency of $250 \mathrm{kHz}$, and the off time of $0.5 \mu \mathrm{s}$. For $\mathrm{TiO}_{2}$ and $\mathrm{Al}_{2} \mathrm{O}_{3}$, the same pulsing configuration was used at a constant power of $800 \mathrm{~W}$. All the depositions were carried out with substrate rotation at a rotation speed of 20 r.p.m. The substrates were kept at floating potential, and no intentional heating was used.

The deposition rates of $\mathrm{Au}$ and $\mathrm{TiO}_{2}$ or $\mathrm{Al}_{2} \mathrm{O}_{3}$ were calculated after depositing thicker layers and determining their thicknesses using a Dektak 150 profilometer. The depositions were carried out in a three-step process, depositing titania (or alumina), $\mathrm{Au}$, and again titania (or alumina). The thickness of each oxide layer was set to about $20 \mathrm{~nm}$. Au layers with thickness corresponding to 1,2 , and $3 \mathrm{~nm}$ were selected. Although $\mathrm{Au}$ does not form a continuous film at such low thickness, the nominal thickness is used to identify the studied samples.

The structure was analysed by X-ray diffraction (XRD) using a Philips (PANalytical) diffractometer with $\mathrm{Co}-\mathrm{K}_{\alpha}$ radiation in grazing incident configuration (GIXRD), with an incident angle of $2^{\circ}$. The XRD parameters, such as the peak position $\left(2 \theta_{0}\right)$, peak intensity, and full width at half maximum (FWHM) were evaluated after fitting the XRD patterns using a Voigt function. The surface morphology of the coatings was analysed by high resolution (HR) field emission gun scanning electron microscopy (FE-SEM) using a Zeiss Leo 1550 microscope. The ImageJ $1.45 \mathrm{~s}$ program was then employed for image analysis. Additional characterization of the Au islands concerning their surface morphology was performed by atomic force microscopy (AFM) using a Veeco-Innova microscope operating in contact mode and using ultra sharp silicon nitride tips (with $2 \mathrm{~nm}$ tip radius). Rutherford backscattering spectroscopy (RBS) experiments were performed with a 5 MV HVEE Tandetron accelerator. ${ }^{16}$ RBS spectra were collected using a $3.035 \mathrm{MeV} \mathrm{He}^{+}$ beam in order to improve the sensitivity to oxygen at the non-Rutherford cross section resonance ${ }^{16} \mathrm{O}(\alpha, \alpha){ }^{16} \mathrm{O}$. The data were acquired simultaneously with two silicon surface barrier detectors located at scattering angles of $170^{\circ}$ with an energy resolution of $16 \mathrm{keV}$ and an ion dose of $10 \mu \mathrm{C}$ per detector. The experimental spectra were fitted using the program RBX. ${ }^{17}$ The optical transmittance of the films was recorded in a UV-Vis-NIR spectrophotometer, using a Shimadzu SolidSpec-3700.

\section{THEORETICAL BACKGROUND}

\section{A. Optical properties of nanocomposite thin films}

The most common way to model the optical properties of a nanocomposite system is to use the concept of effective dielectric function (EDF). One of the most used mean-field theories for the EDF calculation is the Maxwell-Garnett (MG) approximation, which is valid in the low concentration limit (volume fraction of the particles $f \ll 1$, always lower than $0.1^{18}$ ). The MG approach can be improved and extended to higher $f$ by taking into account the dipole-dipole interactions between particles, ${ }^{19}$ a formalism that can be referred to as renormalized MG (RMG) approximation. ${ }^{20}$ The RMG approach was first derived in Ref. 21 and has been generalized to nonspherical particles in Ref. 19.

Considering the classical MG approach, under the mean field approximation, the effective dielectric function $\varepsilon_{\text {eff }}$ of 
the composite system containing a small volume fraction $f$ of separate inclusions on a host medium is given by ${ }^{22}$

$$
\frac{\varepsilon_{e f f}-\varepsilon_{h}}{\varepsilon_{e f f}+2 \varepsilon_{h}}=\frac{4 \pi}{3} N \bar{\alpha}
$$

where $\varepsilon_{h}$ is the dielectric function of the host medium, $N$ $=f / V$ is the particles concentration ( $V$ refers to the particle volume), and $\bar{\alpha}=\sum_{i} \alpha_{i} / 3$ ( $\alpha_{i}$ refers to the polarizability of the individual particle following a principal axis).

An individual spherical particle of radius $a \ll \lambda$ polarized by an external electromagnetic field, without interacting with other particles, is described by the polarizability ${ }^{23,24}$

$$
\alpha_{i}=a^{3} \frac{\varepsilon_{m}-\varepsilon_{h}}{\varepsilon_{m}+2 \varepsilon_{h}},
$$

where $\varepsilon_{m}$ is the dielectric function of the metal inclusion. For ellipsoidal particles with semi axes $a, b$ and $c$, an analogous expression can be found in the quasistatic approximation via introducing geometrical depolarization factors $L_{i}$ along these $\operatorname{axes}^{23,24}$

$$
\alpha_{i}=\frac{a b c}{3} \frac{\varepsilon_{m}-\varepsilon_{h}}{\varepsilon_{h}+L_{i}\left(\varepsilon_{m}-\varepsilon_{h}\right)}, \quad i=1,2,3,
$$

where $\Sigma L_{i}=1$. For spherical particles, $L_{1}=L_{2}=L_{3}=1 / 3$ and the expression (3) resumes to Eq. (2). If the particle possesses axial symmetry, two of the depolarization factors coincide. Prolate ("cigar"-shaped) spheroids, for which $b=c$ and $L_{2}=L_{3}$, can be generated by rotating an ellipse about its major axis and have the following analytical expressions for $L_{1}$ and $L_{2}$ as a function of the eccentricity $e$ :

$$
\begin{gathered}
L_{1}=\frac{1-e^{2}}{e^{2}}\left(-1+\frac{1}{2 e} \ln \frac{1+e}{1-e}\right), \quad e^{2}=1-\frac{b^{2}}{a^{2}}, \\
L_{2}=\frac{1-L_{1}}{2} .
\end{gathered}
$$

Oblate ("pancake"-shaped) spheroids, for which $a=b$ and $L_{1}=L_{2}$, are generated by rotating an ellipse about its minor axis and have the following expressions for $L_{1}$ and $L_{3}$ as a function of $e$ :

$$
\begin{gathered}
L_{1}=\frac{1}{2 e^{2}} \sqrt{\frac{1-e^{2}}{e^{2}}}\left(\frac{\pi}{2}-\tan ^{-1} \sqrt{\frac{1-e^{2}}{e^{2}}}\right)-\frac{1}{2}\left(\frac{1-e^{2}}{e^{2}}\right), \\
e^{2}=1-\frac{c^{2}}{a^{2}}, \\
L_{3}=1-2 L_{1} .
\end{gathered}
$$

The plasmon resonance thus splits into a strongly redshifted long-axis mode (polarization parallel to the long axis) and a slightly blue-shifted short axis mode (polarization perpendicular to the long axis). ${ }^{24}$ Considering incoming light with normal incidence and ellipsoids with one of their axes perpendicular to a plane but with otherwise random orientations, the following simplification can be used for the average polarizability:

$$
\bar{\alpha}=\frac{\alpha_{1}+\alpha_{2}}{2}=\frac{a b c}{6}\left[\frac{\varepsilon_{m}-\varepsilon_{h}}{\varepsilon_{h}+L_{1}\left(\varepsilon_{m}-\varepsilon_{h}\right)}+\frac{\varepsilon_{m}-\varepsilon_{h}}{\varepsilon_{h}+L_{2}\left(\varepsilon_{m}-\varepsilon_{h}\right)}\right] .
$$

The dipole-dipole interaction between the particles renormalizes their average polarizability, which then becomes $^{19}$

$$
\alpha^{*}=\frac{2 \bar{\alpha}}{\kappa}\left[1-\frac{\sqrt{1-\kappa(1-\delta)}}{2}\left(\sqrt{1-\nu}+\frac{\arcsin \nu^{1 / 2}}{\nu^{1 / 2}}\right)\right],
$$

where $\quad \nu=3 \kappa \delta /[1-\kappa(1-\delta)], \quad \kappa=f(4 \pi \bar{\alpha} / 3 V)^{2}, \quad$ and $\delta$ $=\left(\alpha_{\perp}-\alpha_{\|}\right) /\left(\alpha_{\perp}+\alpha_{\|}\right)$is an anisotropy parameter that accounts for the differences in polarizability (between the axis perpendicular $\alpha_{\perp}$ and the axis parallel $\alpha_{\|}$to the axis of symmetry of the spheroid). When $f \rightarrow 0$, then $\alpha^{*} \rightarrow \bar{\alpha}$. The effective dielectric function within the RMG approach is calculated by replacing $\bar{\alpha}$ with $\alpha^{*}$ in Eq. (1).

Once the effective dielectric function $\varepsilon_{\text {eff }}$ is known, the optical properties of the nanocomposite thin films can be determined. For simulating light propagation in planar multilayer thin films, including the effects of multiple internal reflections and interference, the "Transfer Matrix Method"25 was implemented in Mathematica. This allowed calculating the total reflectance $(R)$ and transmittance $(T)$ of the nanocomposites. The absorbance $(A)$ of the system was afterwards calculated through the relation $A=-\log T$.

\section{B. Refractive index sensitivity of LSPR sensors}

Three material parameters can be responsible for changing the plasmon resonance peak as a function of changes in operating temperature and ambient gas atmosphere: ${ }^{8}$ refractive index of the host matrix $\left(n_{h}\right)$, free carrier density of $\mathrm{Au}$ $(N)$, and damping frequency of free carriers in $\mathrm{Au}(\Gamma)$. Gas phase interactions that cause changes in $n_{h}$ and $N$ result predominantly in a shift of the LSPR absorption peak while interactions that modify $\Gamma$ are expected to predominantly create a broadening or narrowing of the peak.

The changes in the refractive index of the metal oxides have been proposed as the primary mechanism causing the shifts in the plasmon resonance peaks position of Au nanoparticles incorporated into a number of different metal oxide matrixes, including $\mathrm{Au}-\mathrm{TiO}_{2}, \mathrm{Au}-\mathrm{WO}_{3}, \mathrm{Au}-\mathrm{NiO}, \mathrm{Au}-\mathrm{CuO}$, and $\mathrm{Au}-\mathrm{Co}_{3} \mathrm{O}_{4} .^{7,26-29}$ Such changes are due to the physisorption or chemisorption of the analyte molecules onto the surface of the metal-oxide and into the porous matrix of the films. ${ }^{7}$ Metal oxides are usually slightly off-stoichiometric resulting in either an excess $\left[\mathrm{M}_{\mathrm{m}+\mathrm{x}} \mathrm{O}\right]$ or a deficiency $\left[\mathrm{M}_{\mathrm{m}-\mathrm{x}} \mathrm{O}\right]$ in metal ions $(\mathrm{M})$ compared to oxygen ions $(\mathrm{O})$, originating either $\mathrm{n}$ - or p-type semiconductor, respectively. ${ }^{30}$ Atmospheric oxygen, for instance, is reported to adsorb preferentially on the surface defects of these oxides, resulting in changes of the conductivity and optical properties according to the $n$ - or p-nature of the oxide vacancies. ${ }^{31}$

Some recent studies suggest that at high temperatures, the variations in the effective electron density of the $\mathrm{Au}$ 
nanoparticles can be the main cause for explaining the SPR peak shifts of some systems. ${ }^{8,32}$ Concerning such cases, it was recently pointed out that the reducing gases that oxidize over metal oxide nanocomposites containing Au nanoparticles should donate electrons to the oxide matrix (that afterwards can interact with $\mathrm{Au}$ ) and not directly to the $\mathrm{Au}$ nanoparticles. ${ }^{31}$ Such changes in the matrix will also likely induce a change in the polarizability, changing its dielectric constant as well. ${ }^{33}$ Thus, for the cases where $N$ seems to play a definite role in the change of the optical property of interest (which seems likely at least for the higher operating temperatures of certain metal oxides), a more complex panorama of interactions is present that requires further studies in order to clearly identify and quantify each individual contribution responsible for the overall observed change in the optical signal.

In this work, the sensitivity of an SPR sensor will be evaluated in terms of refractive index sensitivity, which is valid for many Au-oxide systems operating between room temperature (R.T.) and moderately higher temperatures $\left(300-400^{\circ} \mathrm{C}\right)$. Two main strategies are currently being used to quantify the variations in the SPR peak position: either by calculating the difference in the peak maxima position or by determining the variation in the peak height at a convenient point in the curve. The former strategy will be used here.

The refractive index sensitivity of an SPR sensor with spectral interrogation is defined as ${ }^{34-37}$

$$
S=\frac{d \lambda_{r e s}}{d n_{h}}=\frac{\left(\frac{d \varepsilon_{m}^{\prime}(\lambda)}{d n_{h}}\right)_{\lambda=\lambda_{r e s}}}{\left(\frac{d \varepsilon_{m}^{\prime}(\lambda)}{d \lambda}\right)_{\lambda=\lambda_{\text {res }}}}
$$

where $\varepsilon_{m}^{\prime}$ is the real part of the complex metal dielectric constant $\left(\varepsilon_{m}=\varepsilon^{\prime}{ }_{m}+i \varepsilon^{\prime \prime}{ }_{m}\right)$. If the refractive index of the sensing medium is altered by $d n_{h}$, then the resonance wavelength shifts by $d \lambda_{\text {res }}$. The refractive index sensitivity of the resonance is determined by the same two relations that determine the resonance wavelength $\lambda_{\text {res }}$, the resonance condition and the wavelength dependence of the dielectric function. ${ }^{34}$

Resonance occurs at poles of the polarizability. At wavelengths where the imaginary part of the metal dielectric function is small or slowly varying, the pole of the polarizability is found by setting the real part of the denominator in Eq. (3) to zero. This results in the resonance condition $^{34}$

$$
\varepsilon_{m}^{\prime}\left(\lambda_{\text {res }}\right)=-\frac{1-L}{L} n_{h}^{2},
$$

which determines the value of the real part of the dielectric function at resonance. For the case of a sphere, $L=1 / 3$, and Eq. (11) reduces to the familiar expression $\varepsilon_{m}^{\prime}\left(\lambda_{\text {res }}\right)=-2 \varepsilon_{h}$. The resonance wavelength $\lambda_{\text {res }}$ is complex because the LSP is lossy due to material and radiative losses. Since the complex permittivity of a metal is a holomorphic function, for metals fulfilling $\operatorname{Im}\left\{\lambda_{\text {res }}\right\} \ll \operatorname{Re}\left\{\lambda_{\text {res }}\right\}$, the following relationship between the complex resonant wavelength $\lambda_{\text {res }}$ and the real wavelength of maximum extinction at which extinction peak is observed $\lambda_{\text {peak }}$ holds:

$$
d \lambda_{\text {peak }}=\operatorname{Re}\left\{d \lambda_{\text {res }}\right\}
$$

Thus, the changes in the experimental data can be directly related to the analytical variations. ${ }^{38}$

The LSPR phenomenon originates in the collective excitation of free-electrons of the metal particles, which can be described as a three-dimensional free-electron gas by the Drude theory (the first term of Eq. (21)). The real and imaginary parts of the metal dielectric constant near the resonance are thus given by

$$
\begin{aligned}
\varepsilon_{m}^{\prime}(\omega, R) & =1-\frac{f_{0} \omega_{p}^{2}}{\omega^{2}+\Gamma_{0}^{2}(R)} \\
\text { and } \quad \varepsilon^{\prime \prime}{ }_{m}(\omega, R) & =\frac{f_{0} \omega_{p}^{2} \Gamma_{0}(R)}{\omega^{3}+\Gamma_{0}^{2}(R) \omega} .
\end{aligned}
$$

The previous expressions can be written in terms of wavelength by using the equality $\omega=2 \pi c / \lambda$ (where $c$ is the speed of light in free space). For the real part of the metal dielectric constant, it reads

$$
\varepsilon_{m}^{\prime}(\lambda, R)=1-\frac{f_{0} \omega_{p}^{2}}{(2 \pi c / \lambda)^{2}+\Gamma_{0}^{2}(R)} .
$$

Substituting Eqs. (11) and (14) into Eq. (10) results in the general-shape expression for LSPR sensitivity

$$
S=\frac{n_{h}[(1-L) / L]\left[\left(2 \pi c / \lambda_{\text {res }}\right)^{2}+\Gamma_{0}^{2}(A, R)\right]^{2}}{\omega_{p}^{2}(2 \pi c)^{2} / \lambda_{\text {res }}^{3}} .
$$

The ability of a spectroscopic SPR sensor to resolve small refractive index changes is directly proportional to the refractive index sensitivity and indirectly proportional to the width of the resonant feature. ${ }^{35,36,38,39}$ In order to further infer the sensing potential of the coatings, the following figure of merit can thus be introduced: ${ }^{35,36,38}$

$$
\chi=\left|\frac{S}{w}\right|,
$$

where $S$ is the sensitivity and $w$ is the FWHM of the extinction peak. This figure of merit does not explicitly account for another important factor that is the SPR peak intensity. However, the variations in SPR peak intensity are indirectly accounted by the $w$ term; the narrowing of an SPR peak is usually accompanied by an increase in the peak intensity, and the broadening of an SPR peak is accompanied by a decrease in the peak intensity.

\section{RESULTS}

This section starts with the chemical analysis of the coatings, then describes the structural and morphological analysis, and finally the optical properties and theoretical refractive index sensitivity are evaluated. 


\section{A. Au concentration in the coatings}

The evolution of the elemental composition of the films with Au thickness was studied by RBS. From this analysis, it was possible to determine the atomic density of $\mathrm{Au}$ in the coatings. For the $\mathrm{TiO}_{2}$ case, a total of $8.8 \times 10^{15}$, $1.35 \times 10^{6}$, and $1.82 \times 10^{16} \mathrm{~cm}^{-2}$ were obtained for the 1,2 , and $3 \mathrm{~nm}$ thick Au layer, respectively (Table I). Assuming continuous $\mathrm{Au}$ layers, the corresponding thicknesses would be $0.74,1.74$, and $2.47 \mathrm{~nm}$, taking into account the Au bulk density and molar mass. These values are roughly $20 \%$ lower than the theoretical values, which might be due to errors in the determination of the deposition rate from the thicker $\mathrm{Au}$ film.

\section{B. Structural properties}

The titanium oxide can crystallize in different structures. At ambient conditions, four structures are known: rutile, anatase, brookite, and srilankite. ${ }^{15}$ The most common and stable structure of $\mathrm{TiO}_{2}$ is rutile. ${ }^{40} \mathrm{TiO}_{2}$ thin films with all of the four structures can be synthesized although for deposition temperatures below $150^{\circ} \mathrm{C}$, amorphous $\mathrm{TiO}_{2}$ thin films are usually obtained. Regarding the $\mathrm{Al}_{2} \mathrm{O}_{3}$, it can appear as the thermodynamically stable $\alpha$-alumina as well as crystallized under several other metastable polymorphs. For depositions with substrate temperatures below $300^{\circ} \mathrm{C}$, sputtered alumina coatings are typically amorphous. ${ }^{41}$

For all the $\mathrm{Au} @ \mathrm{TiO}_{2}$ and $\mathrm{Au} @ \mathrm{Al}_{2} \mathrm{O}_{3}$ coatings, no XRD peak concerning any of the $\mathrm{TiO}_{2}$ or $\mathrm{Al}_{2} \mathrm{O}_{3}$ phases was found. This was expected considering the low substrate temperature during depositions. On the other hand, for both systems, $\mathrm{Au}$ was found to be increasingly crystalline with increasing $\mathrm{Au}$ content. In Figure 1, a close up of the XRD spectra concerning the $\mathrm{Au}$ (111) peak for the $\mathrm{Au} @ \mathrm{TiO}_{2}$ and $\mathrm{Au} @ \mathrm{Al}_{2} \mathrm{O}_{3}$ cases is shown. The particle sizes estimated from Scherrer formula ${ }^{42}$ are presented as well.

It can be seen in Figure 1 that for the different oxide systems and Au contents, the XRD peaks become always wider after the encapsulation step. This trend suggests slightly smaller clusters after the top oxide deposition. This apparent diminution in the clusters sizes might be simply the result of the nucleation of smaller Au clusters $(<1-2 \mathrm{~nm})$ that were initially trapped in the oxide defects (being undetectable either by HRSEM or XRD) and that are now able to contribute to the XRD spectra. The possible changes in the morphology of the clusters could also add to the changes in the XRD peak widths. Another more remote possibility is that the bigger clusters consisting of aggregates of two or more smaller clusters get cleaved during the bombardment of the top oxide layer, thus moving the size distribution curve towards the smaller values. It was found that, for all the $\mathrm{Au}$ contents, the clusters are smaller in the $\mathrm{Al}_{2} \mathrm{O}_{3}$ system. This can be explained by the higher surface mobility of $\mathrm{Au}$ on the $\mathrm{TiO}_{2}$ surface.

\section{Surface morphology}

SEM analysis was performed before and after the deposition of the top oxide layer of $\mathrm{TiO}_{2}$ or $\mathrm{Al}_{2} \mathrm{O}_{3}$, Figure 2 and Figure 3, respectively. From Figure 2, it is apparent that the size and shape of the Au nanoparticles strongly depends on the thickness of the sputtered Au layer. When increasing the Au thickness from 1 to $3 \mathrm{~nm}$, the Au clusters shape goes from rather circular to fiber-like and their average particle size increases considerably from 6 to 10 and to $17 \mathrm{~nm}\left(\mathrm{TiO}_{2}\right)$ and from 4 to 7 and to $12 \mathrm{~nm}\left(\mathrm{Al}_{2} \mathrm{O}_{3}\right)$. These three different cases illustrate well the growth modes expected for $\mathrm{Au}$ on oxide surfaces: ${ }^{43-45}$ clusters at $1 \mathrm{~nm}$ thick layer picture corresponds to the end of the first stage of nucleation of nanometric 3D hemispherical Au clusters (Figure 2(a)); at $2 \mathrm{~nm}$, a second stage of lateral growth and some coalescence of the 3D clusters occurs (Figure 2(b)); and at $3 \mathrm{~nm}$, third stage of pronounced coalescence of the 3D clusters takes place (Figure 2(c)). At even higher thicknesses, a vertical growth of the coalesced clusters would finally be observed. By comparing the micrographs of the two oxides, it becomes apparent that the Au clusters are much bigger and the cluster density much lower in the $\mathrm{TiO}_{2}$ case, for all the $\mathrm{Au}$

TABLE I. Surface analysis results of Au nanoparticles over $\mathrm{Al}_{2} \mathrm{O}_{3}$ and $\mathrm{TiO}_{2}$.

\begin{tabular}{|c|c|c|c|c|c|c|}
\hline \multirow{2}{*}{$\frac{\text { Oxide }}{\text { Nominal Au thickness (nm) }}$} & \multicolumn{3}{|c|}{$\mathrm{Al}_{2} \mathrm{O}_{3}$} & \multicolumn{3}{|c|}{$\mathrm{TiO}_{2}$} \\
\hline & 1 & 2 & 3 & 1 & 2 & 3 \\
\hline Ellipse major axis $-\bar{a}(\mathrm{~nm})$ & 5.9 & 9.9 & 16.9 & 6.7 & 12.8 & 22.9 \\
\hline Ellipse minor axis $-\bar{b}(\mathrm{~nm})$ & 3.9 & 6.3 & 9.3 & 4.7 & 8.1 & 12.4 \\
\hline Au area fraction- $-A(\%)$ & 34.2 & 41.7 & 47.1 & 36.0 & 44.1 & 49.7 \\
\hline Particle density $\left(/ \mu \mathrm{m}^{2}\right)$ & 17044 & 8096 & 3622 & 13702 & 5254 & 2110 \\
\hline $\mathrm{Au}$ atomic density $(\mathrm{RBS})\left(/ \mathrm{cm}^{2}\right)$ & $\ldots$ & $\ldots$ & $\ldots$ & $8.8 \times 10^{15}$ & $1.4 \times 10^{16}$ & $1.8 \times 10^{16}$ \\
\hline Cluster's height (RBS/SEM)— $\bar{c}(\mathrm{~nm})$ & 3.7 & 6.7 & 8.4 & 3.3 & 6.2 & 8.1 \\
\hline Average size of the bigger axes (oblate) $-\bar{x}_{a b}(\mathrm{~nm})$ & 4.8 & 7.9 & 12.5 & 5.6 & 10.2 & 16.7 \\
\hline Average size of the smaller axes (prolate) $-\bar{x}_{b c}(\mathrm{~nm})$ & 3.8 & 6.5 & 8.9 & 4.0 & 7.1 & 10.0 \\
\hline Aspect ratio (oblate shape) $-A R_{o b l}$ & 0.76 & 0.84 & 0.67 & 0.59 & 0.60 & 0.48 \\
\hline Aspect ratio (prolate shape) $-A R_{\text {pro }}$ & 0.64 & 0.65 & 0.52 & 0.59 & 0.55 & 0.44 \\
\hline Equivalent diameter of a sphere (nm) & 4.4 & 7.5 & 11.0 & 4.7 & 8.6 & 13.2 \\
\hline Interparticle distance $-D(\mathrm{~nm})$ & 7.8 & 11.6 & 17.4 & 8.9 & 15.0 & 23.8 \\
\hline Au average height (XRD)- $-h^{\prime}(\mathrm{nm})$ & 3.6 & 5.1 & 5.3 & 4.3 & 5.3 & 6.4 \\
\hline Au average height (AFM) $-h(\mathrm{~nm})$ & 2.8 & 3.5 & 3.9 & 3.1 & 3.4 & 3.6 \\
\hline Volume fraction (optical) $-f^{\prime}$ & 0.10 & 0.13 & 0.13 & 0.08 & 0.10 & 0.10 \\
\hline
\end{tabular}


(a)

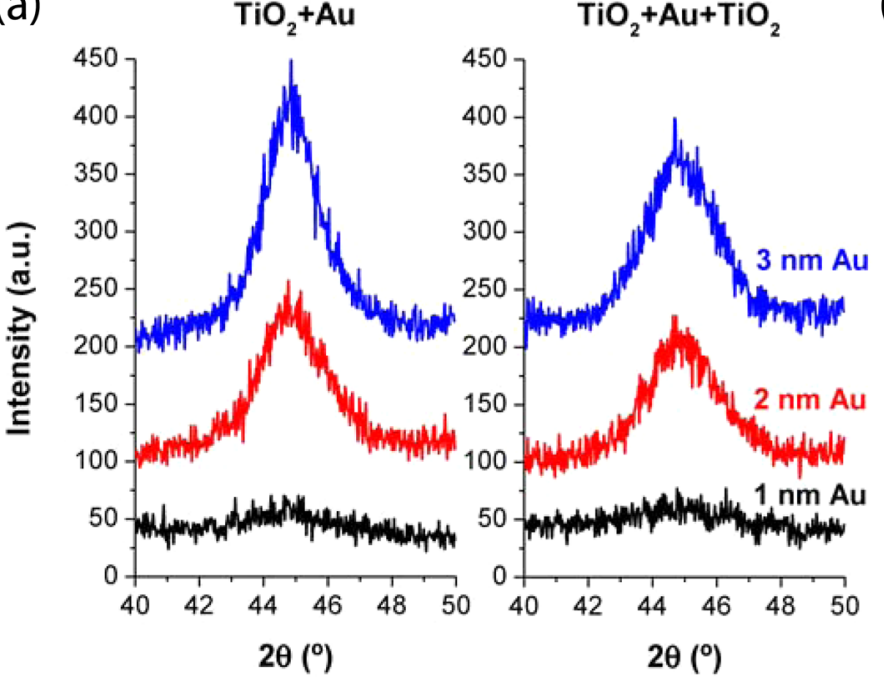

(b)

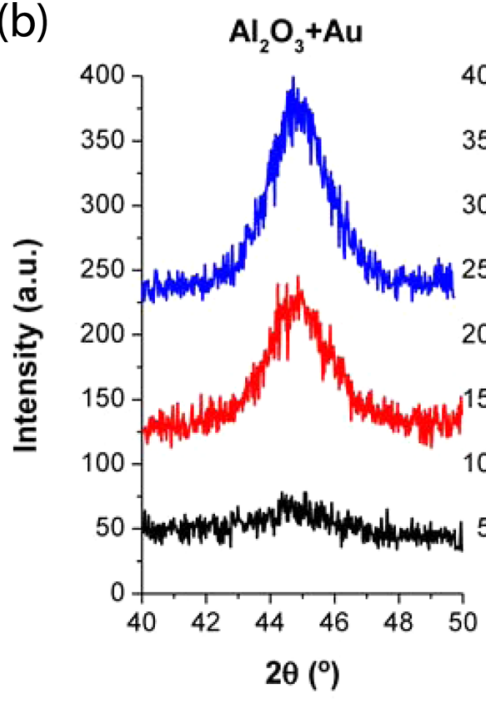

$\mathrm{Al}_{2} \mathrm{O}_{3}+\mathrm{Au}+\mathrm{Al}_{2} \mathrm{O}_{3}$

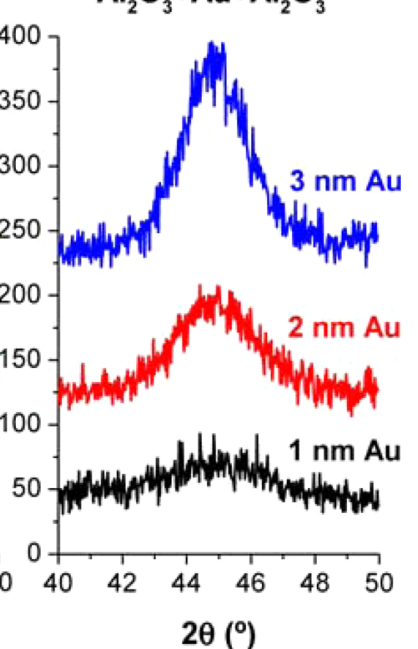

(c)

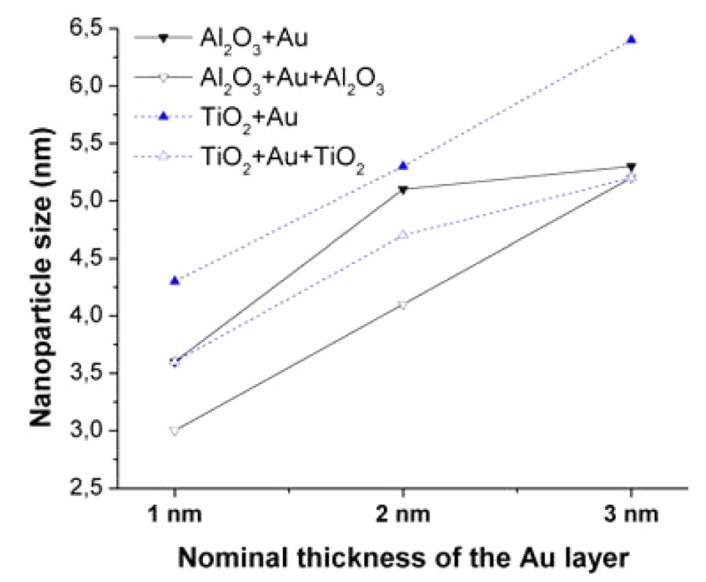

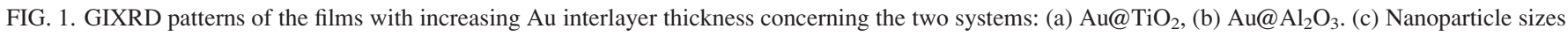
estimated by the Scherrer formula, before (open symbols) and after the final encapsulation (full symbols) step.

concentrations. The higher surface mobility of $\mathrm{Au}$ in the $\mathrm{TiO}_{2}$ explains this difference.

From the FE-SEM micrographs of each nanocomposite film (Figure 2), the Au area fraction, the average major and minor axis of fitted ellipses, the Au mean particle size, and the number density of Au nanoparticles were obtained by image analysis. The results are given in Table I. From the $\mathrm{Au}$ atomic density determined by RBS and the particle density determined by SEM, it was possible to calculate the average clusters height assuming the presence of spheroidal particles. The particle volume of a whole spheroid is defined by

$$
V_{\text {particle }}=\frac{1}{6} \pi a b c
$$

where $a, b$, and $c$ are the equivalent main axes. Knowing the average particle volume through the relation

$$
\bar{V}_{\text {particle }}=\frac{M_{A u}}{\rho_{A u}} \frac{\text { atomic density }}{\text { particle density }},
$$

where $M_{A u}$ is the molar mass in [g/atom], $\rho_{A u}$ is the density in $\left[\mathrm{g} / \mathrm{nm}^{3}\right]$, the atomic density is in $\left[\operatorname{atom} / \mu \mathrm{m}^{2}\right]$ and the particle density is in [number of particles $/ \mu \mathrm{m}^{2}$ ], makes then easy to calculate the average height $c$ of the cluster. Since the deposition conditions used for depositing Au were identical for the two oxides, the Au atomic density measured on $\mathrm{TiO}_{2}$ was used for the $\mathrm{Al}_{2} \mathrm{O}_{3}$ case as well.

The interparticle distance was evaluated with the $\mathrm{Au}$ nanoparticle neighbourhood defined by the Voronoi boundary in the Voronoi diagram of each film. ${ }^{46}$ For both systems, each Au nanoparticle had six neighbours on average. For the hexagonal lattice, the average interparticle separation can be written as ${ }^{45,46}$

$$
D=\frac{\pi^{1 / 2} \bar{x}}{12^{1 / 4} A^{1 / 2}} .
$$

Finally, and for comparison purposes, the diameter of a sphere with an equivalent volume to the spheroid can be calculated through the following equation:

$$
D_{\text {sphere }}=\sqrt[3]{a b c} .
$$

All the results of these calculations are summarized in Table I. In most cases, the calculated clusters heights are close but smaller than the minor lateral dimensions of the 

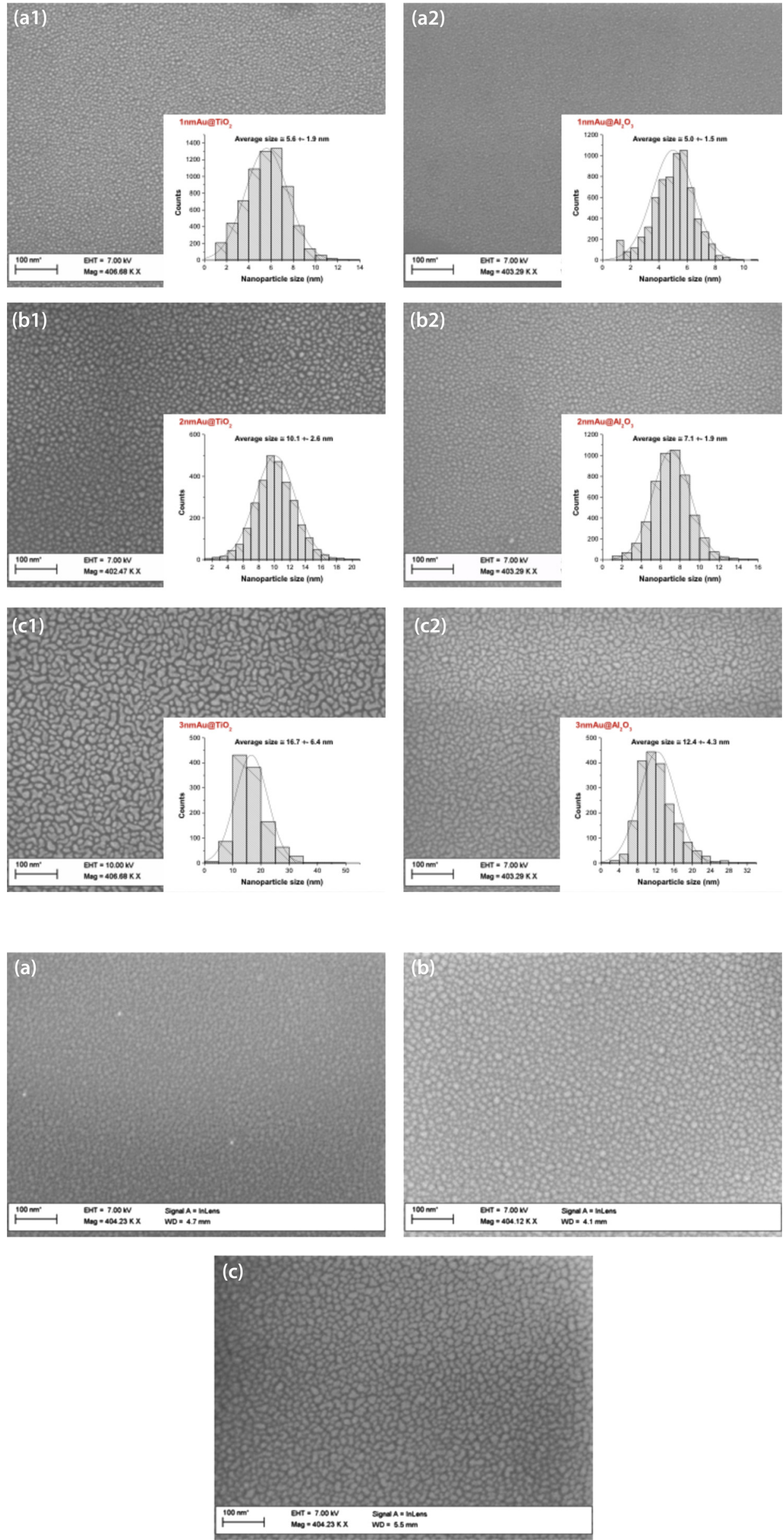

FIG. 2. HRSEM images of the nanocomposites with increasing $\mathrm{Au}$ thickness, for the $\mathrm{TiO}_{2}+\mathrm{Au}$ : (a1) $1 \mathrm{~nm}$, (b1) $2 \mathrm{~nm}$, (c1) $3 \mathrm{~nm}$ and for the $\mathrm{Al}_{2} \mathrm{O}_{3}$ + Au: (a2) $1 \mathrm{~nm}$, (b2) $2 \mathrm{~nm}$, (c2) $3 \mathrm{~nm}$. Histograms representing the Au clusters size distributions are shown in inset.

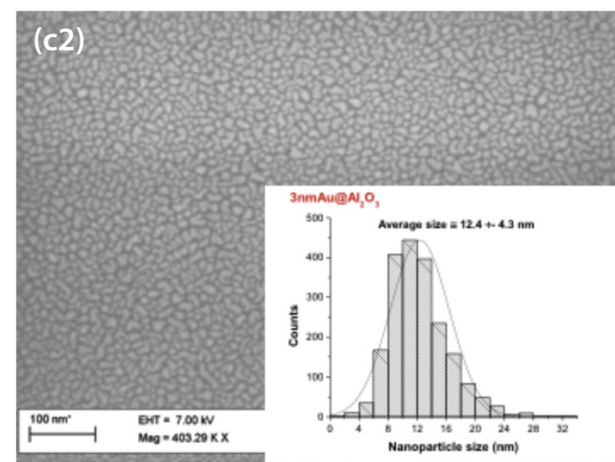

FIG. 3. HRSEM images of the $\mathrm{TiO}_{2}+$ $\mathrm{Au}+\mathrm{TiO}_{2}$ samples with increasing Au interlayer thickness: (a) $1 \mathrm{~nm}$, (b) 2 $\mathrm{nm}$, (c) $3 \mathrm{~nm}$. 
clusters. In order to determine the aspect ratio of the spheroidal particles, an average of either the two bigger axes (oblate) or the two smaller axes (prolate) was considered. Higher aspect ratios (ARs) were achieved for the oblate shapes, suggesting better agreement with experiments.

Additional information about the clusters morphology and its variation with the deposition time was obtained by AFM. From the AFM images of both the Au@ $\mathrm{TiO}_{2}$ and $\mathrm{Au} @ \mathrm{Al}_{2} \mathrm{O}_{3}$ systems, the average height of the deposited clusters was determined (Table I). For both systems, it was found that the clusters heights calculated by AFM were lower than calculated from the RBS/SEM data and even than calculated from XRD. Since the measured AFM image is a convolution of the tip geometry and the geometry of the clusters, lower values for the heights can be measured due to the close proximity of the clusters. On the other hand, the presence of smaller clusters in between the SEM detected clusters could contribute as well to the lower values found for the clusters height. A few line profiles were performed in zones of the samples containing minor scratches (case where the clusters were absent from the oxide surface). The average values for the clusters heights were greater than the averaged ones from the intact zones (the values were closer to the ones obtained from the XRD technique).

In general terms, the data from the different characterization techniques are consistent, especially when considering the small sizes of the nanoparticles being studied.

The current coatings design allows optimization of coatings topography for gas sensing. Due to the presence of $\mathrm{Au}$ nanoparticles with different sizes and shapes in the interlayer and to the chosen thickness of the metal-oxide overcoat, it is possible to attain a top oxide layer with increased roughness and surface area (high density of surface defects) - a topography following the one of the $\mathrm{Au}$ clusters underneath. In Figure 3, the existence of $\mathrm{TiO}_{2}$ caps separated with clear interfaces (corresponding to the zones without $\mathrm{Au}$ ) is observed. The size and shape of these caps closely follows the ones of the Au nanoparticles. Both the reactivity and gas permeability of the thin $\mathrm{TiO}_{2}$ and $\mathrm{Al}_{2} \mathrm{O}_{3}$ films are expected to be enhanced by the present coatings design.

\section{Optical properties}

Optical transmission spectroscopy measurements were carried out in order to study the SPR effect with the $\mathrm{Au} @ \mathrm{TiO}_{2}$ and $\mathrm{Au} @ \mathrm{Al}_{2} \mathrm{O}_{3}$ coatings (Figure 4).
In Figure 4, it is observed that the overall transmission decreases with the increase in the Au thickness. This corresponds to an enhanced absorption due to the higher volume fractions of $\mathrm{Au}$ (Table I). There is also a red-shift and a broadening of the SPR absorption peaks with increasing Au thickness, due to a combined effect of changes in the size and shape of Au nanoparticles as well as in the interparticle distances (Figure 2).

In order to elucidate the connection between the structural and microstructural properties and the extinction signal of the nanocomposite films, a theoretical modelling of the optical properties was performed using the concept of effective dielectric function (EDF).

As an approximation for $\varepsilon_{h}$, the optical constants (refractive index $n$ and extinction coefficient $k$ ) of a pure $\mathrm{Al}_{2} \mathrm{O}_{3}$ and $\mathrm{TiO}_{2}$ thin film (deposited under the same conditions as in the nanocomposites) were estimated numerically from the transmittance results by means of an unconstrained optimization technique described in detail in Ref. 47. These constants were then substituted in the complex expression $\varepsilon_{h}=(n+i k)^{2}$. The thickness of each oxide layer was also estimated with the application of this method. For the pure $\mathrm{Al}_{2} \mathrm{O}_{3}$ and $\mathrm{TiO}_{2}$ films, the refractive index at a wavelength of $550 \mathrm{~nm}$ was 1.68 and 2.43 and the thickness of the thin films was 14 and $16 \mathrm{~nm}$, respectively.

To represent $\varepsilon_{A u}$, an analytical expression determined by Rakic et ll $^{48}$ based on the Brendel-Bormann (BB) model was used. It was shown by the authors that, for $\mathrm{Au}$, the BB model allows better description of experimental data than the more frequently used Drude-Lorentz (DL) model, especially where the lowest-laying interband transitions occurs. The BB model accounts for the transitions within the conduction band, by using a Drude term, as well as for the interband transitions from lower-lying bands into the conduction band or from the conduction band into higher unoccupied levels, by using a superposition of an infinite number of oscillators. The resulting expression for the dielectric function of the metal is the following: ${ }^{48}$

$$
\begin{aligned}
\varepsilon(\omega)= & 1-\frac{\Omega_{p}^{2}}{\omega^{2}+i \Gamma_{0} \omega} \\
& +\sum_{j=1}^{k} \frac{1}{\sqrt{2 \pi} \sigma_{j}} \int_{-\infty}^{+\infty} \exp \left[-\frac{\left(x-\omega_{j}\right)^{2}}{2 \sigma_{j}^{2}}\right] \\
& \times \frac{f_{j} \omega_{p}^{2}}{\left(x^{2}-\omega^{2}\right)+i \omega \Gamma_{j}} d x,
\end{aligned}
$$
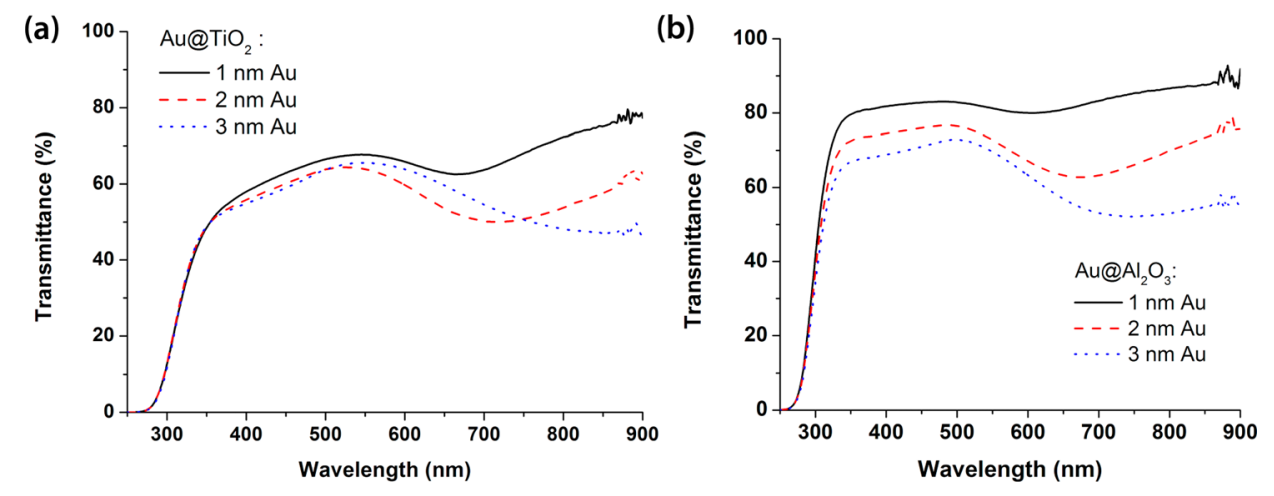

FIG. 4. Transmission spectra of the (a) $\mathrm{Au} @ \mathrm{TiO}_{2}$ and (b) $\mathrm{Au} @ \mathrm{Al}_{2} \mathrm{O}_{3}$ samples on glass with increasing Au interlayer thickness. 
where $\omega$ is the frequency of light, $\omega_{p}$ is the plasma frequency of the metal, $\Omega_{p}=\sqrt{f_{0}} \omega_{p}$ is the plasma frequency associated with intraband transitions with oscillator strength $f_{0}$ and damping constant $\Gamma_{0}$, while $k$ is the number of BB oscillators used to interpret the interband part of the spectrum with frequency $\omega_{j}$, strength $f_{j}$, lifetime $1 / \Gamma_{j}$, and line shape parameter $\sigma_{j}$. The number of harmonic oscillators per frequency interval is determined by a Gaussian function. In the present work, the analytical solution of the second term of Eq. (21) given in Ref. 48 was used for the calculations.

In order to account for the intrinsic size effects of the nanoparticles, the BB model was modified by correcting the plasmon damping parameter $(\Gamma)$ according to the relation ${ }^{49}$

$$
\Gamma=\Gamma_{0}+\frac{A \nu_{F}}{R},
$$

where $\Gamma_{0}$ is the relaxation constant of the bulk metal (given in Ref. 48), $A$ is a phenomenological parameter that is a function of the geometry, of the order of unity, and which in practice is adjusted to provide the best fit of the data, ${ }^{50} \nu_{F}$ is the Au Fermy velocity $\left(1.39 \times 10^{15} \mathrm{~nm} / \mathrm{s}\right)$, and $R$ is the radius of the nanoparticle (in $\mathrm{nm}$ ).

One way of estimating the volume fraction $f$ of metal islands from two-dimensional pictures is by using the concept of "optical thickness" $t_{\text {opt }}$ as in terms of effective depolarization factors. An appropriate definition would be to use $^{45}$

$$
t_{\text {opt }}=\frac{4}{3} D,
$$

where $D$ is the average particle's center-to-center distance. The volume fraction follows then from the relation ${ }^{45}$

$$
f=\frac{t}{t_{\text {opt }}}
$$

where $t$ is the nominal thickness of the film.

The results of these calculations were compiled into Table I.

The simulations of the optical absorbance spectra were performed considering a stack of three layers on a glass substrate. The stack consisted of a top oxide layer, followed by an intermediate nanocomposite layer and another bottom oxide layer with characteristics identical to the first layer. Different parameters were varied in order to achieve a good fit to the experimental data, namely, the Au volume fraction $f$, the phenomenological parameter $A$, the average diameter $2 R$, the shape (spherical, oblate, and prolate) and aspect ratio $A R$ of the clusters, and the thickness of each layer $d_{i}$. The simulated absorbance spectra are presented in Figure 5, and their corresponding fitting parameters are listed in Table II. The simulated spectra were divided into two major groups A and $\mathrm{B}$, following the two types of spheroidal particles that were tested, oblate and prolate, respectively.

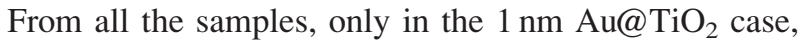
it was possible to consistently describe the optical absorbance spectrum by means of using perfectly spherical shapes in the simulations $(A R=1)$. For all the other cases, both the oblate and the prolate shapes were successfully implemented in the simulations. The main differences in parameters between the two spheroidal shapes were found in the volume fraction and in the aspect ratio of the clusters. They were higher for the prolate shapes, and this difference in values became more pronounced with the increase in the nominal Au thickness. The size effect parameters and particles sizes used to correct the Au dielectric function were similar in both cases. When comparing simulation results between oxide systems, it was clear that higher $A R \mathrm{~s}$ and lower $f \mathrm{~s}$ were needed for the $\mathrm{TiO}_{2}$ case, irrespectively of the shape being considered.

When simulating the optical response of the metallic spheroidal nanoparticles, two of the axes were considered to be parallel to the thin film surface. For the particular case of the prolate shape, this resulted in the appearance of two distinct resonances, the less intense blue-shifted one being somewhat masked for the case of higher $A R \mathrm{~s}$, as shown in Figure 5(a), but becoming evident for lower $A R \mathrm{~s}$, as it is seen in Figures 5(b) and 5(c). In the experimental data, the higher energy resonance is clearly absent, which again suggests the better agreement of the oblate shape with the experiment. Still, for the two $2 \mathrm{~nm}$ Au samples and the $3 \mathrm{~nm}$ $\mathrm{Au} @ \mathrm{TiO}_{2}$ sample, it was possible to satisfactorily simulate the absorbance spectra by assuming the presence of prolate shapes.

Overall, the simulations performed considering particles with oblate shapes (group A) showed better (very good) agreement with the experimental data of both the oxides, even for the $3 \mathrm{~nm}$ Au samples, case where the particles were more irregular shaped. Aditionally, the simulations using oblate shapes gave as well Au volume fraction values closer to the ones determined from SEM images using Eq. (24).

The results from the simulations concerning the clusters heights (given in this case by the parameter $2 R$ ) seem to agree better with the XRD results than with the combined RBS/SEM results (which give higher values). When estimating previously the cluster heights using RBS and SEM data, all the Au atoms were considered to be in the SEM-detected clusters. Both the optical and the structural results suggest that even after the encapsulation step, there are still smaller $\mathrm{Au}$ atoms $(<1-2 \mathrm{~nm})$ present in the coatings.

\section{E. Sensitivity of the LSPR sensors}

In Figure 6, the sensitivities and figure of merit calculated for the different coatings based on the previous simulation results are presented. The shape that showed better agreement with experiment, i.e., the oblate shape (simulation group A) was used in the calculations.

Figure 6(a) shows that, for both oxides, there is an increase in the sensitivity with the nominal thickness of Au. This is mainly due to the decrease in the depolarization factor $L$ (Table II) with the $\mathrm{Au}$ increase. When comparing results between oxides, overall the $\mathrm{Al}_{2} \mathrm{O}_{3}$ matrix shows lower sensitivity values, despite the lower depolarization values achieved in the simulation. This is mainly due the lower refractive index of the $\mathrm{Al}_{2} \mathrm{O}_{3}$. The only exception is in the $2 \mathrm{~nm} \mathrm{Au}$ layer, case where the difference in the 
(a1)

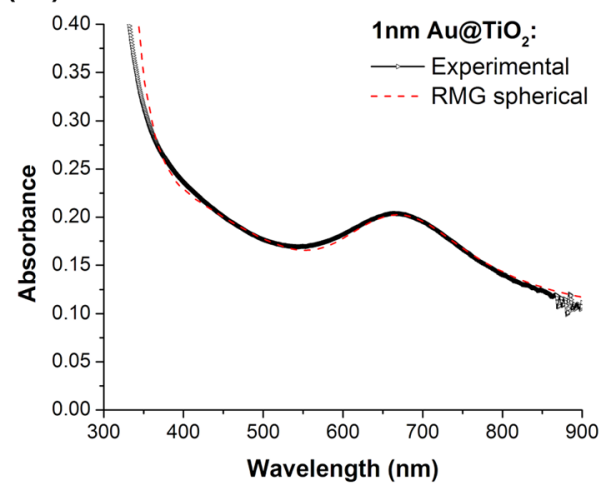

(b1)

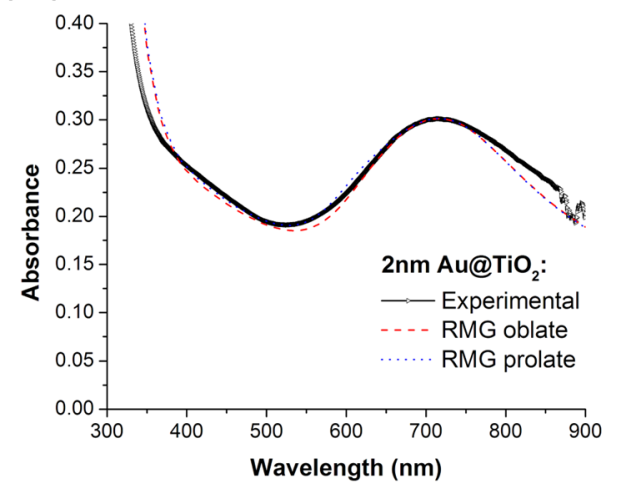

(c1)

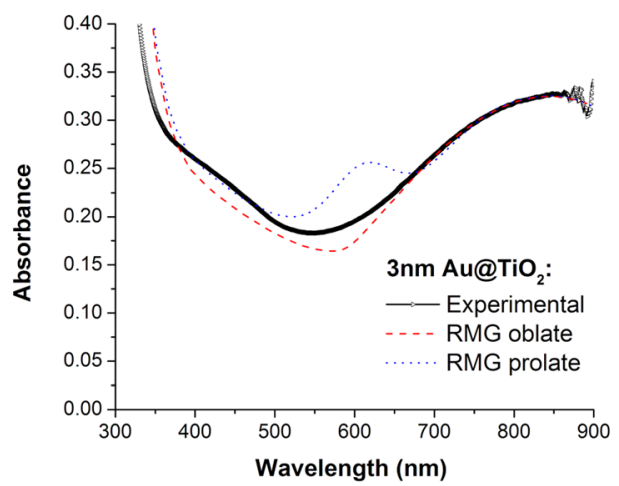

(a2)

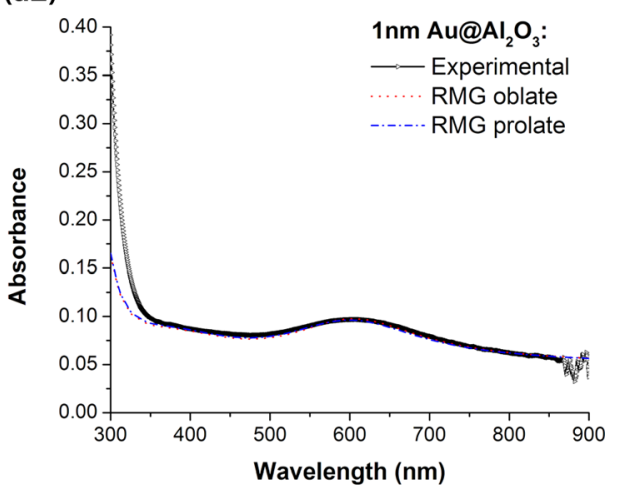

(b2)

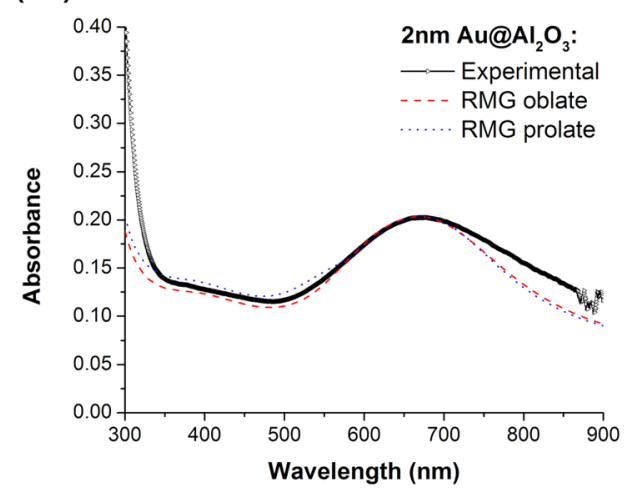

(c2)

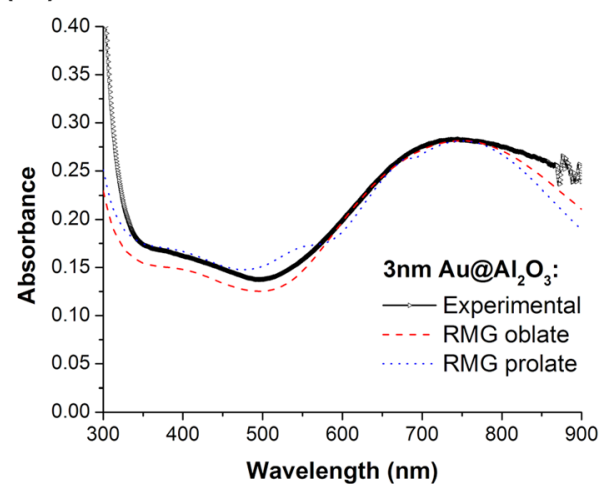

FIG. 5. Theoretical and experimental absorbance spectra of the $\mathrm{Au} @ \mathrm{TiO}_{2}$ and $\mathrm{Au} @ \mathrm{Al}_{2} \mathrm{O}_{3}$ samples on glass substrate with increasing $\mathrm{Au}$ interlayer thickness: (a) $1 \mathrm{~nm}$, (b) $2 \mathrm{~nm}$, (c) $3 \mathrm{~nm}$.

TABLE II. Parameters used for the calculation of spectra presented in Figure 5, namely, aspect ratio $(A R)$ and diameter of the clusters ( $2 R)$, size effect parameter $(A)$, volume fraction $(f)$, and layer thickness $(d)$. The calculated depolarization factors $(L)$ are also shown.

\begin{tabular}{|c|c|c|c|c|c|c|c|c|c|c|c|}
\hline \multirow[b]{2}{*}{ Simulation } & \multirow[b]{2}{*}{ Oxide system } & \multirow[b]{2}{*}{ Au thickness (nm) } & \multirow[b]{2}{*}{ Particle shape } & \multirow[b]{2}{*}{$A R$} & \multirow[b]{2}{*}{$L$} & \multirow[b]{2}{*}{$A$} & \multirow[b]{2}{*}{$2 R(\mathrm{~nm})$} & \multirow[b]{2}{*}{$f$} & \multicolumn{3}{|c|}{ Layer thickness $d(\mathrm{~nm})$} \\
\hline & & & & & & & & & Top oxide & Nanocomposite & Bottom oxide \\
\hline \multirow[t]{6}{*}{ A } & \multirow[t]{3}{*}{$\mathrm{TiO}_{2}$} & 1 & Spherical & 1 & 0.333 & 0.75 & 3.6 & 0.066 & 16 & 9 & 16 \\
\hline & & 2 & Oblate & 0.83 & 0.308 & 0.9 & 4.2 & 0.092 & 14 & 16.7 & 14 \\
\hline & & 3 & Oblate & 0.5 & 0.236 & 1 & 5.7 & 0.110 & 14.5 & 17.2 & 14.5 \\
\hline & \multirow[t]{3}{*}{$\mathrm{Al}_{2} \mathrm{O}_{3}$} & 1 & Oblate & 0.78 & 0.298 & 0.8 & 2.4 & 0.073 & 14 & 10 & 14 \\
\hline & & 2 & Oblate & 0.53 & 0.244 & 0.9 & 4 & 0.105 & 12 & 16.5 & 12 \\
\hline & & 3 & Oblate & 0.4 & 0.206 & 1 & 5.4 & 0.120 & 10 & 21 & 10 \\
\hline \multirow[t]{6}{*}{ B } & \multirow[t]{3}{*}{$\mathrm{TiO}_{2}$} & 1 & Spherical & 1 & 0.333 & 0.75 & 3.6 & 0.066 & 16 & 9 & 16 \\
\hline & & 2 & Prolate & 0.84 & 0.288 & 0.9 & 4.3 & 0.095 & 14 & 17.2 & 14 \\
\hline & & 3 & Prolate & 0.62 & 0.219 & 1 & 5.7 & 0.125 & 14.5 & 17.6 & 14.5 \\
\hline & \multirow[t]{3}{*}{$\mathrm{Al}_{2} \mathrm{O}_{3}$} & 1 & Prolate & 0.81 & 0.280 & 0.8 & 2.4 & 0.077 & 14 & 10.2 & 14 \\
\hline & & 2 & Prolate & 0.62 & 0.219 & 0.9 & 4.2 & 0.120 & 12 & 18 & 12 \\
\hline & & 3 & Prolate & 0.53 & 0.186 & 1 & 5.6 & 0.145 & 10 & 22.5 & 10 \\
\hline
\end{tabular}


(a)

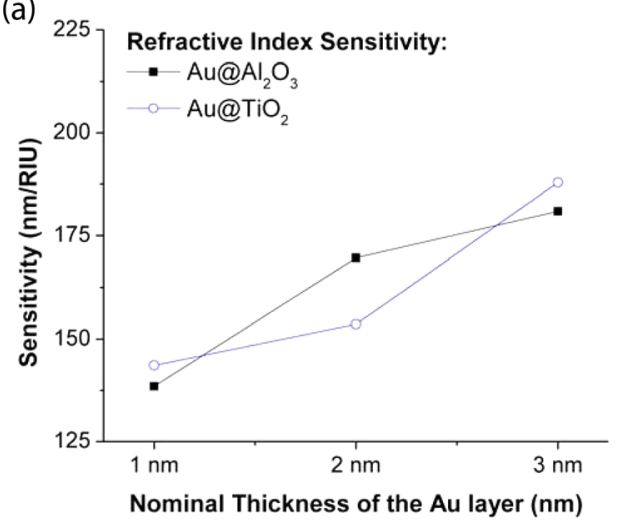

(b)

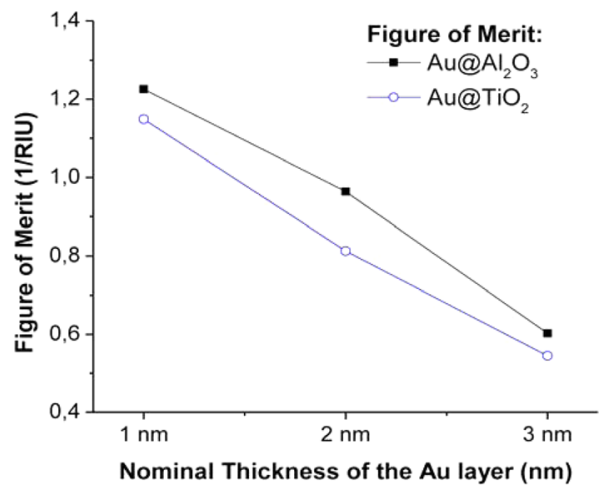

FIG. 6. (a) Refractive index sensitivities and (b) figure of merit determined for the different coatings, considering the simulation data $\mathrm{A}$ (oblate shapes). The first point in the $\mathrm{TiO}_{2}$ series corresponds to spherical shape. depolarization factors is more pronounced. For the 1 and $3 \mathrm{~nm}$ Au cases, this difference in the depolarization factors is attenuated and thus the higher refractive index of $\mathrm{TiO}_{2}$ allows higher sensitivities to be achieved. Interestingly, the figure of merit (Figure 6(b)) shows the opposite trend of the sensitivity, decreasing with the Au thickness. This is due to the pronounced increase in the widths of the SPR peaks with the thickness, originating less sharp SPR features.

\section{THEORETICAL CONSIDERATIONS}

When studying the SPR peaks-mainly shape and position-three major impacting factors concerning the nanoparticles can be identified. An increase in the size of the nanoparticles leads mostly to an increase in the intensity and a narrowing of the SPR peaks (up to a certain size). The increase of dipole-dipole interactions due to reduced interparticle distances leads to a red-shift and a broadening of the SPR peaks. And, finally, the presence of elongated nanoparticles can lead to strongly red-shifted and also broader SPR peaks. ${ }^{20,51}$ A proper analysis of the impact of the cluster's radius, volume fraction, and aspect radius on the refractive index sensitivity is thus desirable in order to allow optimizing the coatings for better sensing performance.

From Eq. (15), it is evident that $S$ increases with $\Gamma_{0}$ and $n_{h}$ and decreases with $L$ and $\lambda_{\text {res }}$. However, the phenomenological parameter $A$, the clusters radius $R$, and the clusters volume fraction $f$ also influence indirectly the refractive index sensitivity. Higher $A$ and smaller $R$ give rise to higher $\Gamma_{0}$ values, which directly increases $S$, but only by a limited quantity. On the other hand, for higher $\Gamma_{0}$ values, $\lambda_{\text {res }}$ is slightly blueshifted, giving as well a small positive contribution to $S$. In practice, however, since higher $\Gamma_{0}$ values promote an increase in the width of the SPR features, they can be detrimental for sensing experiments. It is thus desirable to have clusters presenting minimum intrinsic size effects. This occurs approximately for sizes greater than $30 \mathrm{~nm} .{ }^{50}$ On the other hand, the excitation of higher order multipoles, giving rise to additional damped resonance features in the optical spectra, should be avoided. This implies that the Au clusters should have sizes below $70-80 \mathrm{~nm}^{50}$ The sensitivity is increased for matrices with higher refractive indexes $n_{h}$, despite $\lambda_{\text {res }}$ being also increased with $n_{h}$. The resonance wavelength is strongly affected by $f$. An increase in $f$ will greatly increase $\lambda_{\text {res }}$ (due to the increased dipole-dipole interactions), which results in lower $S$ values. The SPR peak widths are also increased by $f$.
To better understand and visualize the combined effect of the volume fraction and the refractive index of the matrix on the refractive index sensitivity of the films, a threedimensional analysis of the sensitivity was made. In this analysis, the following steps were performed: (i) simulating the absorbance spectra of different sets of coatings with different $n_{h}$ (varying from 1.5 to 2.5 ) and $f$ (varying from 0.01 to 0.15 ), (ii) extracting the SPR peak parameters (peak maxima and FWHM), (iii) fitting linear functions of the resonance wavelength and of the SPR peak width to the sets of data, and (iv) substituting these functions in the expressions of $S$ and $X$. The goal is to identify best parameters for a practical experiment.

Considering spherical particles, and setting $A=0.8$ and $R=15 \mathrm{~nm}$, the following images in Figure 7 were obtained.

It becomes apparent that $S$ is always greater for greater $n_{h}$ and that in general terms $S$ is smaller for smaller $f$. This suggests that it is desirable to have coatings containing $\mathrm{Au}$ volume fractions below $0.05-0.07$ (depending on the type of matrix). This optimum $f$ value should roughly correspond to
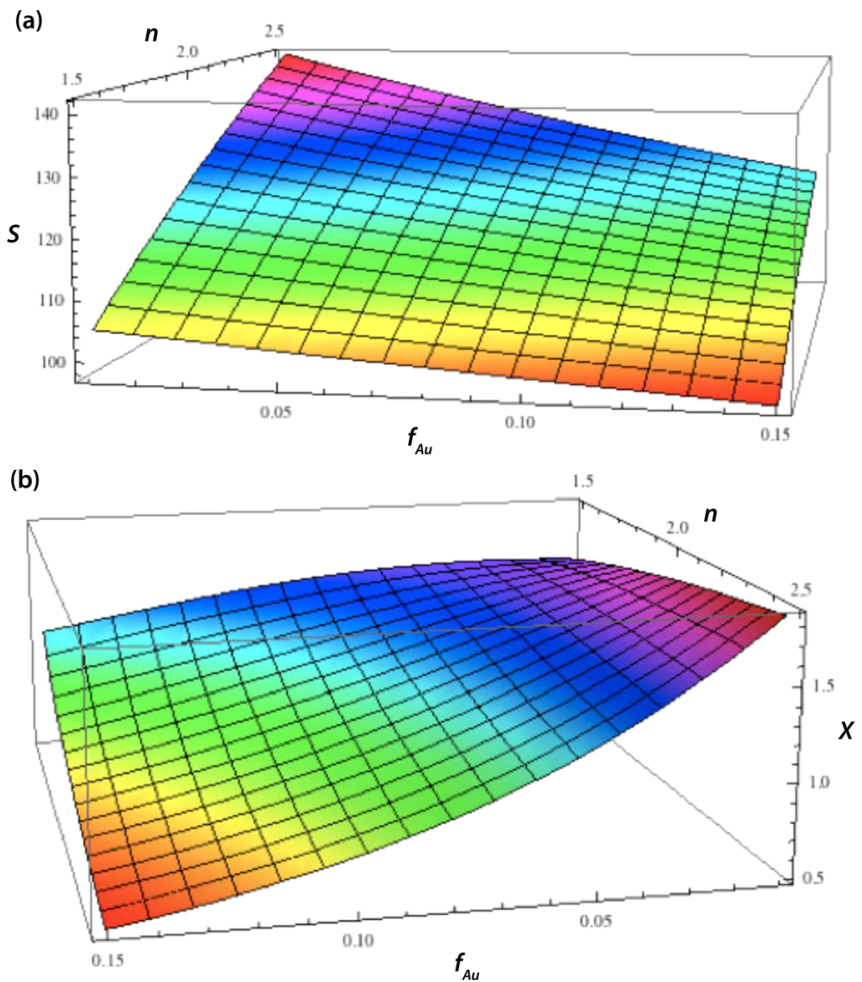

FIG. 7. (a) Refractive index sensitivities and (b) figure of merit calculated for the range of parameters $n_{h} \in[1.5-2.5]$ and $f \in[0.01-0.15]$. 


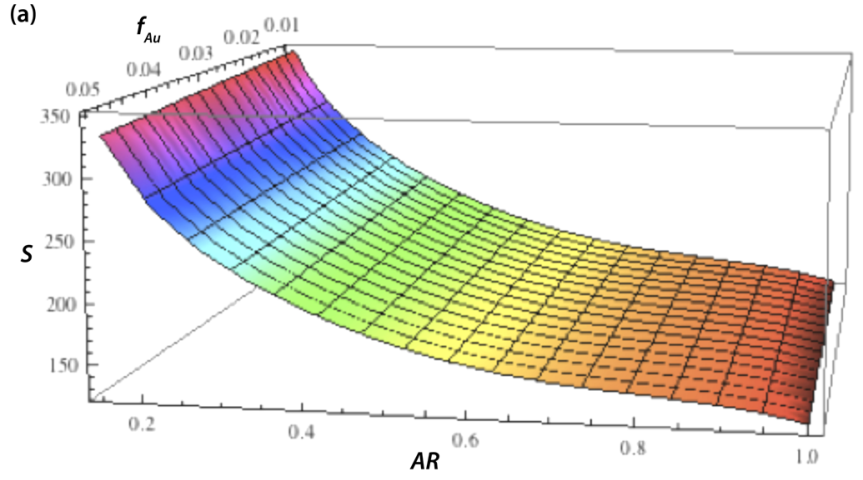

(b)

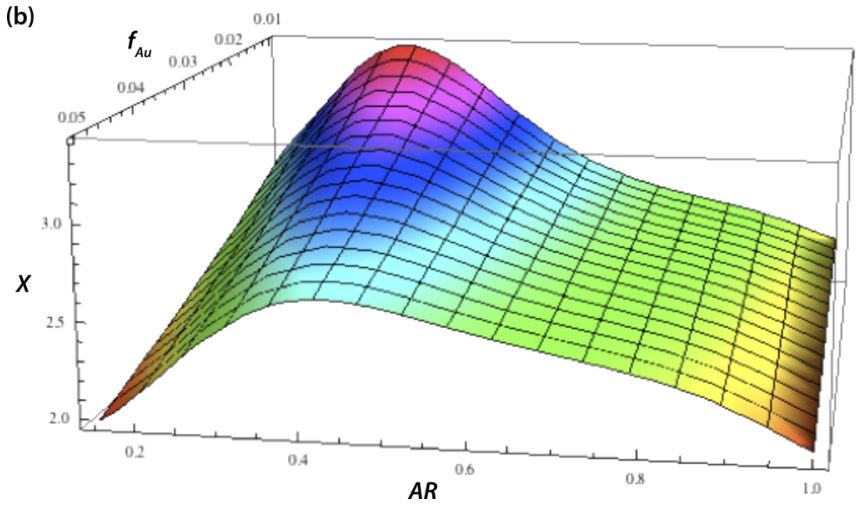

FIG. 8. (a) Refractive index sensitivities and (b) figure of merit calculated for the oblate shape considering the range of parameters $f \in[0.01-0.05]$ and $A R \in[1-0.15]$.

the case where the cluster's separation distances are greater than the clusters diameters for which the dipole-dipole interactions starts to be negligible. Considering $30 \mathrm{~nm}$ particles displaced in a hexagonal lattice, from Eq. (19), the optimum interparticle distance $D$ of $60 \mathrm{~nm}$ could be accomplished for an area fraction $A$ of $23 \%$. If in addition the expression for volume fraction given by Eq. (24) is employed, for a Au thickness of $3 \mathrm{~nm}$, the resulting volume fraction would be 0.04. Such an arrangement of clusters on a surface can be achieved, for instance, by applying substrate heating on conventional sputtering ${ }^{52}$ or by using a cluster source for depositing the metal nanoparticles with the desired size and distribution $^{53}$ during the Au clusters deposition step.

The major factor influencing $S$ is, however, the depolarization factor $L$; when decreasing $L, S$ increases exponentially. $L$, in turn, diminishes when the aspect ratio $A R$ of the spheroidal particle is decreased. In order to understand how the $A R$ of each prolate and oblate shape in the end affects the refractive index sensitivity, an additional three-dimensional analysis was performed, varying $f$ (between 0.01 and 0.05 ) and $A R$ (between 1 and 0.15 ), for the two shapes being considered (oblate and prolate). The $n_{h}$ value was set to 2 . For spheroidal particles, the influence of $n_{h}$ on $S$ is almost negligible when compared to $L$. As in the previous study, the size effect parameters were set to $A=0.8$ and $R=15 \mathrm{~nm}$. The results of the simulations for the oblate and prolate shapes can be seen in Figure 8 and Figure 9, respectively.

The results show that the sensitivities of spheroidal particles increase almost exponentially when decreasing the $A R$, as expected due to the decrease in $L$. The sensitivity values were only slightly affected by $f$, being higher for lower $f$. For
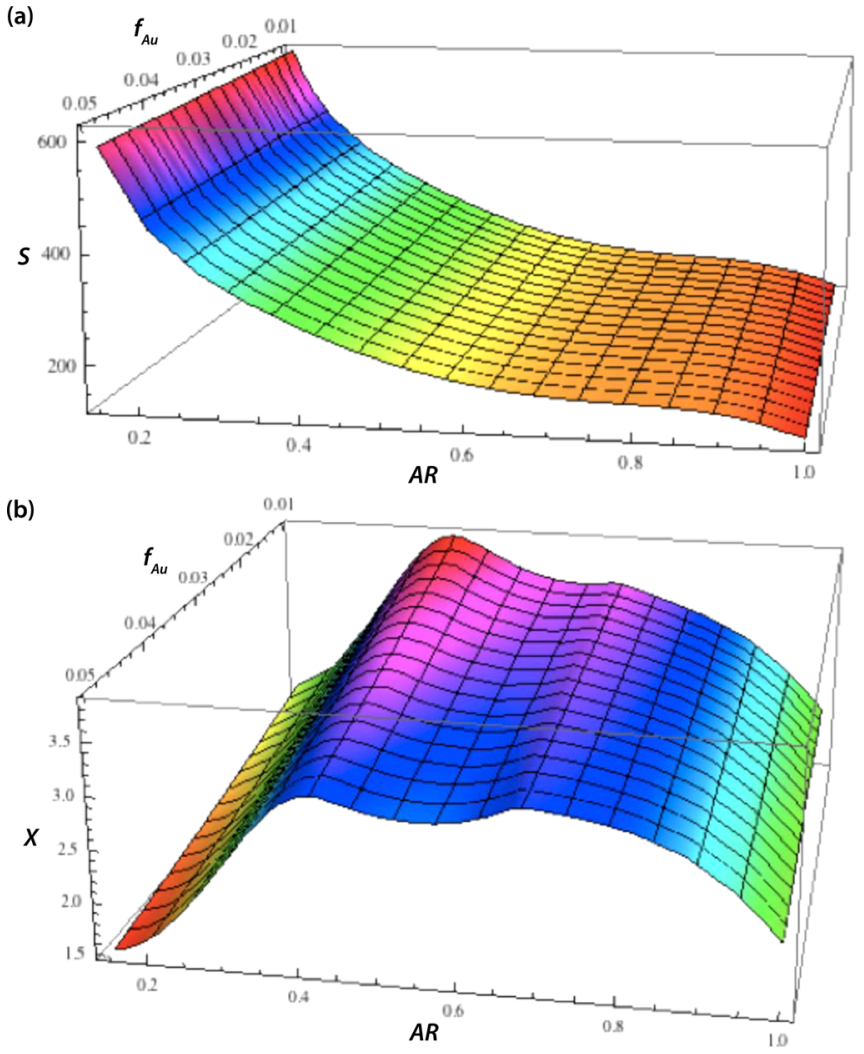

FIG. 9. (a) Refractive index sensitivities and (b) figure of merit calculated for the prolate shape considering the range of parameters $f \in[0.01-0.05]$ and $A R \in[1-0.15]$.

the oblate shape, a maximum $S$ value of 360 was achieved for the lowest studied $A R$ of 0.15 . For the prolate shape, the $S$ values were almost twice as higher than in the oblate shape, and a maximum value of 650 was achieved. The figure of merit values were however similar in both cases. Despite the higher $S$ values of the prolate shape, the widths of their red-shifted resonances are also greatly increased, almost cancelling the benefit of increased $S$. The lower $f$ values allowed higher $X$ values to be reached, but now in a more pronounced way than with $S$. This was due to the increase in the SPR peak widths with $f$. The optimum $A R$ values were found to be 0.35 for the oblate shape (corresponding to a $X$ value of 3.4 ) and 0.42 for the prolate shape (corresponding to a $X$ value of 3.9 ). The deposition by sputtering of the prolate particles with the suggested characteristics seems currently difficult; however, the configuration of oblate clusters can be achieved, for instance, with the application of substrate temperature during the Au clusters deposition step. ${ }^{54}$

\section{CONCLUSIONS}

The simple minimalist design for LSPR refractive index nanosensors that was tested shows excellent flexibility concerning the materials used and permits an easy deposition process.

For both $\mathrm{TiO}_{2}$ and $\mathrm{Al}_{2} \mathrm{O}_{3}$, an increase in the $\mathrm{Au}$ interlayer thickness led to a broadening and a red-shift of the SPR peaks. This was due to a combined effect of increased interparticle interactions and decreased circularity of the particles. The 
coatings containing higher amounts of Au showed higher refractive index sensitivities. The figure of merit was however lower due to the increased width of the resonance peak.

Since the SPR intensity increases with the size of the nanoparticles, and the SPR peaks are narrower in absence of intercoupling, bigger particles with higher interparticle distances are desired to optimize the current design. In order to achieve this configuration in the future, some energetic process (e.g., substrate heating) or a cluster source can be used for depositing the Au interlayers/clusters.

Au nanoparticles of $30 \mathrm{~nm}$ with interparticle distances greater than $60 \mathrm{~nm}$ were considered in simulations of refractive index sensitivities and figure of merit values of spheroidal particles with different aspect ratios. Our results show that higher sensitivities (almost twice as much) can be achieved for prolate particles when comparing to the oblate shapes and that an aspect ratio of 0.42 is optimal when the sensitivities and the peak widths are considered for analysis. For the oblate shapes, the optimum aspect ratio of 0.35 was found.

\section{ACKNOWLEDGMENTS}

This research was sponsored by FEDER funds through the program COMPETE, Programa Operacional Factores de Competitividade, by national funds through FCT, Fundação para a Ciência e a Tecnologia, under the Projects PEstC/EME/UI0285/2013 and CENTRO-07-0224-FEDER-002001 (Mais Centro SCT_2011_02_001_4637), and through the Spanish Ministry of Science and Innovation by Projects FUNCOAT CSD2008-00023 and AIB2010PT-00241. R.E.G. wishes also to thank the MCINN for the financial support within the Ramón y Cajal programme. Funding by the European Community through Project Nano4Color is gratefully acknowledged.

${ }^{1}$ N. M. Figueiredo, C. Louro, R. Escobar Galindo, A. Climent-Font, and A. Cavaleiro, Surf. Coat. Technol. 206, 2740 (2012).

${ }^{2}$ R. B. M. Schasfoort and A. J. Tudos, Handbook of Surface Plasmon Resonance (The Royal Society of Chemistry, 2008).

${ }^{3}$ D. A. Stuart, A. J. Haes, C. R. Yonzon, E. M. Hicks, and R. P. Van Duyne, IEE Proc.-Nanobiotechnol. 152, 13 (2005).

${ }^{4}$ H. Deng, D. Yang, B. Chen, and C.-W. Lin, Sens. Actuators B 134, 502 (2008).

${ }^{5}$ G. Sirinakis, R. Siddique, I. Manning, P. H. Rogers, and M. A. Carpenter, J. Phys. Chem. B 110, 13508-13511 (2006).

${ }^{6}$ P. R. Ohodnicki, C. Wang, and M. Andio, Thin Solid Films 539, 327-336 (2013).

${ }^{7}$ M. G. Manera, J. Spadavecchia, D. Buso, C. Fernández, G. Mattei, A. Martucci, P. Mulvaney, J. Pérez-Juste, R. Rella, L. Vasanelli, and P. Mazzoldi, Sens. Actuators B 132, 107-115 (2008).

${ }^{8}$ P. R. Ohodnicki, M. P. Buric, T. D. Brown, C. Matranga, C. Wang, J. Baltrus, and M. Andio, Nanoscale 5, 9030-9039 (2013).

${ }^{9}$ A. J. Haes, S. Zou, G. C. Schartz, and R. P. Van Duyne, J. Phys. Chem. B 108, 109 (2004).

${ }^{10}$ B. Karunagaran, P. Uthirakumar, S. J. Chung, S. Velumani, and E.-K. Suh, Mater. Charact. 58, 680 (2007).

${ }^{11}$ E. Hutter and J. H. Fendler, Adv. Mater. 16, 1685 (2004).

${ }^{12}$ S. H. Cho, S. Lee, D. Y. Ku, T. S. Lee, B. Cheong, W. M. Kim, and K. S. Lee, Thin Solid Films 447-448, 68 (2004).

${ }^{13}$ A. G. Silva, N. Bundaleski, A. M. Moutinho, and O. M. Teodoro, Appl. Surf. Sci. 258, 2006 (2012).

${ }^{14}$ G. Eranna, B. C. Joshi, D. P. Runthala, and R. P. Gupta, Crit. Rev. Solid State Mater. Sci. 29, 111 (2004).
${ }^{15}$ Y. Li, S. Zhang, Y. Liu, T. P. Chen, T. Sritharan, and C. Xu, J. Nanosci. Nanotechnol. 9, 4116-4120 (2009).

${ }^{16}$ A. Climent-Font, F. Pászti, G. García, M. T. Fernández-Jiménez, and F. Agulló, Nucl. Instrum. Methods Phys. Res., Sect. B 219-220, 400 (2004).

${ }^{17}$ E. Kotai, Nucl. Instrum. Methods Phys. Res., Sect. B 85, 588 (1994).

${ }^{18}$ M. Quinten, Optical Properties of Nanoparticle Systems - Mie and Beyond (Wiley-Vch Verlag GmbH \& Co., Wheinheim, 2011).

${ }^{19}$ M. I. Vasilevskiy, Phys. Status Solidi B 219, 197 (2000).

${ }^{20}$ M. Torrell, R. Kabir, L. Cunha, M. I. Vasilevskiy, F. Vaz, A. Cavaleiro, E. Alves, and N. P. Barradas, J. Appl. Phys. 109, 074310 (2011).

${ }^{21}$ R. G. Barrera, G. Monsivais, and W. L. Mochan, Phys. Rev. B 38, 5371 (1988).

${ }^{22}$ U. Kreibig and M. Vollmer, Optical Properties of Metal Clusters (Springer, Berlin, 1995).

${ }^{23}$ K. D. Sattler, Handbook of Nanophysics 3: Nanoparticles and Quantum Dots (CRC Press, USA, 2011).

${ }^{24}$ C. F. Bohren and D. R. Huffman, Absorption and Scattering of Light by Small Particles (John Wiley \& Sons, USA, 1983).

${ }^{25}$ O. S. Heavens, Optical Properties of Thin Solid Films (Dover Publications, New York, 1991).

${ }^{26}$ M. Ando, R. Chabicovsky, and M. Haruta, Sens. Actuators B 76, 13-17 (2001).

${ }^{27}$ M. Ando, T. Kobayashi, S. Iijima, and M. Haruta, Sens. Actuators B 96, 589-595 (2003).

${ }^{28}$ M. Ando, T. Kobayashi, and M. Haruta, Catal. Today 36, 135-141 (1997).

${ }^{29}$ D. Yang, B. Chen, S. Nikumb, C.-H. Chang, and C.-W. Lin, in Proc. SENSORCOMM (2008), pp. 378-383.

${ }^{30} \mathrm{P}$. Kofstad, Non-Stoichiometric, Diffusion and Electrical Conductivity in Binary Metal Oxides (Wiley, New York, 1972).

${ }^{31}$ E. D. Gaspera, M. Guglielmi, A. Martucci, L. Giancaterini, and C. Cantalini, Sens. Actuators B 164, 54-63 (2012).

${ }^{32}$ N. A. Joy, C. M. Settens, R. J. Matyi, and M. A. Carpenter, J. Phys. Chem. C 115, 6283-6289 (2011).

${ }^{33}$ G. Dharmalingam, N. A. Joy, B. Grisafe, and M. A. Carpenter, Beilstein J. Nanotechnol. 3, 712-721 (2012).

${ }^{34}$ M. M. Miller and A. A. Lazarides, J. Phys. Chem. B 109, 21556-21565 (2005).

${ }^{35}$ K. Lodewijks, W. Van Roy, G. Borghs, L. Lagae, and P. Van Dorpe, Nano Lett. 12, 1655-1659 (2012).

${ }^{36}$ T. Chung, S.-Y. Lee, E. Y. Song, H. Chun, and B. Lee, Sensors 11, 10907-10929 (2011).

${ }^{37}$ B. Brian, B. Sepúlveda, Y. Alaverdyan, L. M. Lechuga, and M. Kall, Opt. Express 17, 2015-2023 (2009).

${ }^{38} \mathrm{P}$. Kvasnicka and J. Homola, Biointerphases 3, FD4-FD11 (2008).

${ }^{39}$ J. Homola, Surface Plasmon Resonance Based Sensors (Springer, Berlin, 2006).

${ }^{40}$ J. Haines and J. N. Léger, Physica B 192, 233 (1993).

${ }^{41}$ J. M. Schneider, W. D. Sproul, R. W. J. Chia, M.-S. Wong, and A. Matthews, Surf. Coat. Technol. 96, 262-266 (1997).

${ }^{42}$ M. Birkholz, Thin Film Analysis by X-Ray Scattering (WILEY-VCH Verlag GmbH \& Co. KGaA, Weinheim, 2006).

${ }^{43}$ L. Zhang, R. Persaud, and T. E. Madey, Phys. Rev. B 56, 10549 (1997).

${ }^{44}$ F. Ruffino and M. G. Grimaldi, J. Appl. Phys. 107, 104321 (2010).

${ }^{45}$ S. Norrman, T. Andersson, and C. G. Granqvist, Phys. Rev. B 18, 674-695 (1978).

${ }^{46}$ D.-G. Kim, Y. J. Lee, N. Koshizaki, and Y. D. Kim, J. Ceram. Process. Res. 9, 158-161 (2008).

${ }^{47}$ E. G. Birgin, I. Chambouleyron, and J. M. Martínez, J. Comput. Phys. 151, 862 (1999).

${ }^{48}$ A. D. Rakic, A. B. Djurišic, J. M. Elazar, and M. L. Majewski, Appl. Opt. 37, 5271 (1998).

${ }^{49}$ Y.-H. Lin, J.-C. Hsu, Y. Ding, and P. W. Wang, Opt. Rev. 16, 400 (2009).

${ }^{50} \mathrm{D}$. Sarid and W. Challener, Modern Introduction to Surface Plasmons Theory, Mathematica Modeling, and Applications (Cambridge University Press, New York, 2010).

${ }^{51}$ S. Christke, C. Katzer, V. Grosse, F. Schmidl, G. Schmidl, W. Fritzsche, J. Petschulat, T. Pertsch, and M. Rettenmayr, Opt. Mater. Express 1, 890 (2011).

${ }^{52}$ G. Celep, E. Cottancin, J. Lermé, M. Pellarin, L. Arnaud, J. R. Huntzinger, J. L. Vialle, and M. Broyer, Phys. Rev. B 70, 165409 (2004).

${ }^{53}$ A. N. Banerjee, R. Krishna, and B. Das, Appl. Phys. A 90, 299-303 (2008).

${ }^{54}$ F. Cosandey, L. Zhang, and T. E. Madey, Surf. Sci. 474, 1-13 (2001). 